\title{
Validation of Sterilization Methods by Means of Gas Sensors and Spore-based Biosensors
}

\author{
J. Arreola $^{1,2}$, J. Oberländer, ${ }^{1,2}$, F. Vahidpour ${ }^{1}$, M. Keusgen ${ }^{2}$, M. J. Schöning ${ }^{1,3}$ \\ ${ }^{1}$ Institute of Nano- and Biotechnologies (INB), Aachen University of Applied Sciences, Heinrich- \\ Mußmann-Str. 1, 52428, Jülich, Germany \\ ${ }^{2}$ Institute of Pharmaceutical Chemistry, Philipps-University Marburg, Marbacher Weg 6-10, 35032 \\ Marburg, Germany \\ ${ }^{3}$ Institute of Complex Systems 8 (ICS-8), Forschungszentrum Jülich, Wilhelm-Johnen-Straße, 52428, \\ Jülich, Germany \\ schoening@fh-aachen.de
}

\begin{abstract}
:
In aseptic food industry, hydrogen peroxide is widely used as sterilization agent. The state-of-the-art methods used for the validation of sterilization procedures are microbiological tests, where highly resistant spores are exposed to the sterilant under investigation. The logarithmic kill rate of the spores is determined and the sterilization can be subsequently assured. However, this is tedious and slow; the results can be obtained at the earliest after 24 hours (usually even after 72 hours, see e.g., count reduction test). For this reason, rapid and efficient sterilization methods are needed. In this work, we present two different sensors to validate sterilization procedures: a calorimetric gas sensor for monitoring hydrogen peroxide concentrations and a spore-based biosensor to evaluate the viability of the spores.
\end{abstract}

Key words: sterilization, hydrogen peroxide, gas sensor, biosensor, spores

\section{Introduction}

Hydrogen peroxide $\left(\mathrm{H}_{2} \mathrm{O}_{2}\right)$ is one of the most used sterilants in aseptic packaging systems for the food industry [1]. The efficacy of $\mathrm{H}_{2} \mathrm{O}_{2}$ is mainly influenced by its temperature, concentration and exposure time. Microbiological tests are carried out to validate and control the effectiveness of such sterilization agents. In these tests, microbiological spores are chosen as a probe due to their high resistance to the sterilants. As the spores are exposed to the sterilization method, their viability is highly decreased and the success of the sterilization can be guaranteed. Although, these microbiological procedures are well-stablished and reliable, they are quite slow and time-consuming. Therefore, the development of novel validation sterilization methods is desirable.

Lately, two different sensors were suggested to monitor the sporicidal effectiveness of hydrogen peroxide, namely a calorimetric $\mathrm{H}_{2} \mathrm{O}_{2}$ gas sensor [2] and a spore-based biosensor [3]. The former can measure the hydrogen peroxide concentration, whereas the latter is able to evaluate the viability of the spores (e.g., through morphological changes). Nonetheless, for the spore-based biosensor, the interaction between the morphology of the spores with hydrogen peroxide and the sensor's signal is still undergoing research. As a result, in the present work, the evaluation of different gaseous $\mathrm{H}_{2} \mathrm{O}_{2}$ concentrations and the physical, chemical and electrical parameters of two different spores (Bacillus atrophaeus DSM 675, Bacillus subtilis DSM 402) will be investigated.

\section{Materials and methods}

i) Calorimetric gas sensor and spore-based biosensor. The calorimetric gas sensor consists of a differential setup of two meander structures serving as temperature sensors. One temperature sensor is catalytically activated and the other is passivated. The temperature difference between them is measured and correlates to the present $\mathrm{H}_{2} \mathrm{O}_{2}$ concentration, as introduced in [2]. Moreover, the spore-based biosensor is composed of a differential setup of interdigitated electrodes (IDE), on one IDE microbiological spores are immobilized, the second serves as a reference. The impedance 
can be measured at the different stages of the sterilization process and it can be correlated to the viability of the spores [3].

ii) Sterilization. The sterilization processes were performed in a sterilization test rig resembling the gaseous $\mathrm{H}_{2} \mathrm{O}_{2}$ procedures used in aseptic filling machines for the food industry; with predefined process conditions such as gas temperature of $240{ }^{\circ} \mathrm{C}$ and $\mathrm{H}_{2} \mathrm{O}_{2}$ concentrations up to $8 \% \mathrm{v} / \mathrm{v}$.

iii) Impedimetric measurements. The sporebased biosensors were analyzed with a precision LCR meter (Agilent Technologies, E4980 A).

iv) Spore suspension. The strain of $B$. atrophaeus DSM 675 and the strain of $B$. subtilis DSM 402 were purchased from DSMZ. The suspensions were prepared as stated in [4]. A spore load of at least $10^{6}$ spores per chip was used.

\section{Results and Discussion}

The sterilization process was monitored by a calorimetric $\mathrm{H}_{2} \mathrm{O}_{2}$ gas sensor as seen in Fig. 1 . The upper part shows the temperature plots of the catalytically activated and passivated temperature sensors. The lower part shows the differential signal and the present $\mathrm{H}_{2} \mathrm{O}_{2}$ concentration.

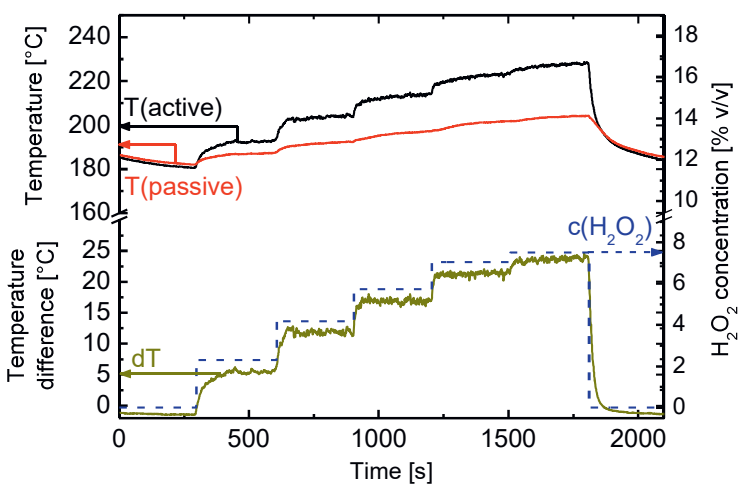

Fig. 1. Sterilization process conditions monitored by a calorimetric $\mathrm{H}_{2} \mathrm{O}_{2}$ gas sensor.

In addition, the sterilization process was also evaluated by a spore-based biosensor with two different spores of Bacillus atrophaeus and Bacillus subtilis (Fig. 2). The upper line (black) of the plot shows the sensor's signal without spores. After the spore immobilization, the impedance decreased and due to the sterilization it further decreased. This impedance difference (spores and $\mathrm{H}_{2} \mathrm{O}_{2}$ gas sterilization) can be correlated with the spore viability.

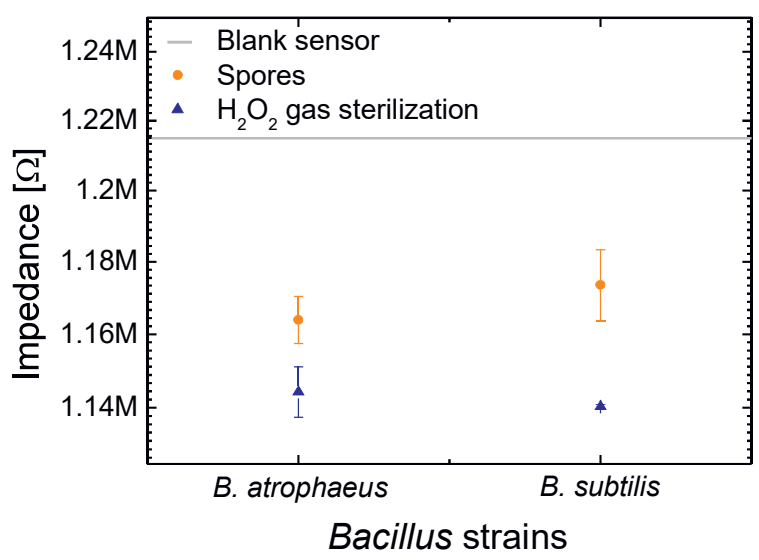

Fig. 2. Evaluation of a sterilization process with gaseous $\mathrm{H}_{2} \mathrm{O}_{2}$ with a spore-based biosensor utilizing different spores of Bacillus atrophaeus and Bacillus subtilis.

\section{Conclusions}

Two independent sensor systems for the evaluation of sterilization processes were introduced; ongoing research is working on the combination of both systems. The process conditions were monitored by a calorimetric $\mathrm{H}_{2} \mathrm{O}_{2}$ gas sensor. Whereas, the sterilization impact on microbiological spores was detected by an impedimetric electrochemical sensor. The capability of the spore-based biosensor to evaluate commonly-used sterilization methods (e.g. autoclave, $\mathrm{O}_{2}$ plasma) will be presented at the conference.

\section{References}

[1] I.A. Ansari and A.K. Datta, Food and Bioproducts Processing 81, 57-65 (2003); doi: $10.1205 / 096030803765208670$

[2] P. Kirchner, J. Oberländer, H.-P. Suso, G. Rysstad, M. Keusgen, M. J. Schöning, Food Control 31, 530-538 (2013); doi: 10.1016/j.foodcont.2012.11.048

[3] J. Oberländer, M. Mayer, A. Greeff, M. Keusgen, M. J. Schöning, Biosensors and Bioelectronics 104, 87-94 (2018); doi: 10.1016/j.bios.2017.12.045

[4] J. Arreola, J. Oberländer, M. Mätzkow, M. Keusgen, M. J. Schöning, Electrochimica Acta 241, 237-243 (2017); doi:

10.1016/j.electacta.2017.04.157

\section{Acknowledgments}

The authors would like to thank J. Bongaerts for supporting the microbiological tests. This project was funded by the BMBF, Germany, Project: "ImpediPack" (Fund. No.: 03FH012I3). 